\title{
CHIRAL EFFECTIVE FIELD THEORIES WITH EXPLICIT SPIN 3/2 DEGREES OF FREEDOM-A STATUS REPORT
}

\author{
THOMAS R. HEMMERT \\ Physik Department T39, TU München, \\ James-Franck-Straße, D-85747 Garching, Germany \\ Email: themmert@physik.tu-muenchen.de
}

Recent developments in calculations of low energy nucleon properties utilizing effective chiral field theories with explicit spin $3 / 2$ matter fields are addressed.

\section{Introduction}

The chiral symmetry of QCD is spontaneously broken at low energies, giving rise to 3 [8] Goldstone Bosons for the case of 2 [3] light quark flavors. In the following, we will concentrate on a world with only 2 light flavors; all other quark degrees of freedom are taken to be infinitely heavy. We identify the 3 Goldstone Boson degrees of freedom with the physical pions, which therefore owe their small (but finite) mass to the additional explicit breaking of $\mathrm{SU}(2)_{L} \times \mathrm{SU}(2)_{R}$ chiral symmetry due to the "small" masses of the up, down quarks. Chiral Perturbation Theory is a successful effective field theory that parameterizes the interactions among these Goldstone Bosons (in the presence of external fields) in the most general form, based solely on the symmetries of the underlying lagrangian of QCD. If this were all, this theory would obviously be not very interesting for the audience of an NSTAR conference. However, very general principles tell us also how these Goldstone bosons interact with "matter"-fields, even in the presence of additional external sources/fields. For an overview on calculations involving Goldstone Bosons + matter fields I refer to the recent review of Ref 1 . Here, I will focus on "matter" that consists of spin $1 / 2$ fermions (Nucleons) and their spin $3 / 2$ resonance partners (Delta(1232)), constraining myself again to a world of 2 light flavors. In particular, I will discuss the role of Delta resonances in microscopic calculations of the anomalous magnetic moments of the Nucleon, the impact of Deltas on the isovector Pauli form factor of the Nucleon and the problems one faces if one wants to calculate the isovector Nucleon-Delta transition form factors. Further topics of recent interest/activity, like the (reduced?) screening of Delta(1232) generated paramagnetism in the isoscalar Nucleon magnetic polarizability $\beta_{M}^{(s)}$ 目 or the impact of Deltas on the momentum-dependence of the generalized spin-polarizabilities of the Nucleon 3 cannot be covered here. 


\section{Chiral calculations and Power-counting}

For systematic calculations with chiral effective field theories one needs a procedure to construct the most general effective chiral lagrangian that contains all possible terms allowed by chiral symmetry, as well as (subsets of) PCT constraints. In addition that tells us, which ones of the plethora of possible diagrams and non-linear vertex structures have to be taken into account if one performs a calculation up to a given order. In the following, I will show results obtained in 2 different chiral effective field theories: SU(2) HBChPT - which constitutes a non-relativistic theory with only pion, Nucleon degrees of freedom and powercounting $\mathcal{O}\left(p^{n}\right)$ - and $\mathrm{SU}(2) \mathrm{SSE}$ - which contains pion, Nucleon and Delta degrees of freedom and power-counting $\mathcal{O}\left(\epsilon^{n}\right)$. For details I have to refer to the literaturet.

Here, I only want to point out that in chiral effective field theories like SSE small parameter, there exists some degree of freedom as to how one organizes the power-counting. The example I want to discuss here concerns the leading $\gamma N \Delta$ vertex, which traditionally is written in the (SU(6) quark-model inspired) form

$$
\mathcal{L}_{\gamma N \Delta}^{(2)}=\frac{b_{1}}{2 M_{0}} \bar{T}_{j}^{\mu} i f_{+\mu \nu}^{j} S^{\nu} N_{v}+\text { h.c. . }
$$

I do not want to discuss this operator structure in detail, the important point to observe is that the dimension-less coupling $b_{1}$ is assumed to scale with a large baryon mass scale $M_{0}$, boosting this structure to $\mathcal{O}\left(\epsilon^{2}\right)$ (i.e. NLO) in the SSE lagrangian. Several reasons - for example the resulting large value for the coupling $b_{1} \sim 7.7$, the failure of $\mathrm{SU}(6)$ symmetry considerations to predict the full strength of the $\gamma N \Delta$ transition, etc.- - have compelled us to propose a slight modification for this operator:

$$
\mathcal{L}_{\gamma N \Delta}^{(1)}=\frac{c_{V}}{\Delta_{0}} \bar{T}_{j}^{\mu} i f_{+\mu \nu}^{j} S^{\nu} N_{v}+\text { h.c. } .
$$

Due to the small mass scale $\Delta_{0}$ in the denominator, the leading $\gamma N \Delta$ transition operator is now a part of the $\mathcal{O}(\epsilon)$ (i.e. LO) SSE lagrangian, leading (in some cases) to a substantial reordering of the chiral expansion. Interesting consequences of this rescaled vertex of Eq.2 2 will be discussed in the following sections.

${ }^{a}$ Furthermore, one needs to specify, how many light quark flavors one wants to consider, whether one utilizes non-relativistic or relativistic chiral effective field theory and which regularization procedure (consistent with the symmetries) one wants to employ. 


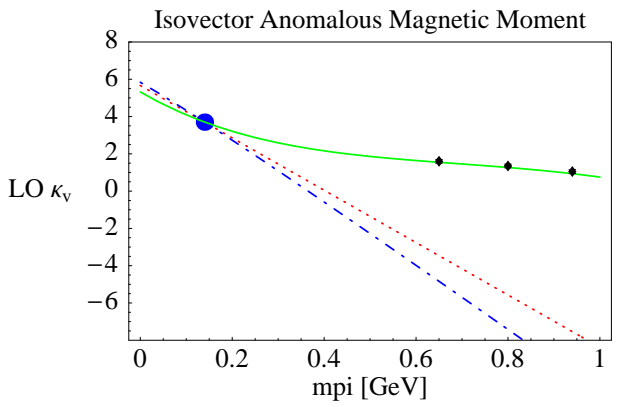

Figure 1. Isovector anomalous magnetic moment of the Nucleon (in Nucleon magnetons) and its dependence on the effective mass of the pion. The physical value at $m_{\pi}=140 \mathrm{MeV}$ is $=3.7[\mathrm{n} . \mathrm{m}]$. Also shown are 3 (quenched) lattice QCD data points of Leinweber et al. 6 ; the curves are explained in the text.

\section{Anomalous magnetic moments of the Nucleon}

Figure 11 shows the leading one-loop order (LO) results obtained in 3 different calculations utilizing chiral effective field theories for the anomalous isovector magnetic moment of the Nucleon $\kappa_{v}$ (in Nucleon magnetons [n.m.]), defined via

$$
\kappa_{v}=\mu_{\text {proton }}-\mu_{\text {neutron }}-1[\text { n.m. }]
$$

and plotted as a function of the pion mass $m_{\pi}$. The dotted curve shows the LO HBChPT result of

$$
\kappa_{v}^{(3)}=\kappa_{v}^{(0)}-\frac{g_{A}^{2} M_{N}}{4 \pi F_{\pi}^{2}} m_{\pi}+\mathcal{O}\left(p^{4}\right),
$$

with the free parameter $\kappa_{v}^{0}$ fixed in such a way that $\kappa_{v}^{(3)}$ reproduces the physical value of $\kappa_{v}^{\text {(phys.) }}=3.7$ [n.m.] at $m_{\pi}=140 \mathrm{MeV} . g_{A}$ denotes the axial coupling constant of the Nucleon with mass $M_{N}$, and $F_{\pi}$ is the pion-decay constant. All of these quantities are taken at their physical values, as any implicit quark-mass dependence constitutes an effect of higher order in the chiral expansion. Figure 1 also shows that it is not possible to connect the LO HBChPT result with the results of a quenched lattice QCD calculation by Leinweber et al. 9 , employing effective pion masses of $600 \mathrm{MeV}$ and higher. A few years ago 6 , the LO influence of explicit Delta(1232) degrees of freedom on the vector (and axial-vector) current of the Nucleon was analyzed utilizing the Small Scale Expansion of Ref.t. As can be clearly seen by the dashed 


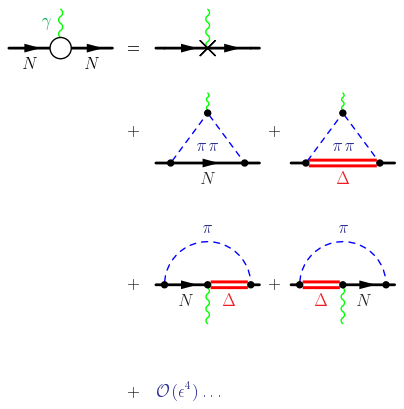

Figure 2. Diagrams contributing to the anomalous magnetic moments of the Nucleon to leading one-loop order in the modified Small Scale Expansion.

curve in Figure 1, there is only a small modification due to the explicit spin $3 / 2$ degrees of freedom for small pion mass. Still no satisfying connection to the lattice points can be obtained.

We now discuss the results of a new calculationt of the isovector anomalous magnetic moment which utilizes the rescaled leading $\gamma N \Delta$ vertex of Eq.2 discussed in the previous section. The diagrams taken into account to leading one-loop order (i.e. $\mathcal{O}\left(\epsilon^{3}\right)$ ) in the now modified Small Scale Expansion are shown in Fig.2. The experts in the audience will notice that there are 2 additional diagrams (in the last row) of Fig.2 compared to the calculation of 6 . The result of this calculation is shown by the full curve in Fig.1. It contains 3 free parameters $-\kappa_{v}^{0}$, the isovector anomalous magnetic moment of the Nucleon in the chiral limit, $c_{V}$, the new leading order $\gamma N \Delta$ coupling constant introduced in Eq.22, and one additional (quark-mass dependent) higher order $\gamma N N$ coupling constant $E_{1}(\lambda)$, which also serves as a counterterm to absorb new divergences coming in due to the 2 extra diagrams. All other parameters can be fixed from known low energy quantities. In Fig 1, we have fit the 3 parameters $\kappa_{v}^{0}, c_{\mathrm{H}}, E_{1}(1 \mathrm{GeV})$ to the 3 (quenched) lattice QCD data points of Leinweber et al. , but not to the $\kappa_{v}$ value at the physical pion mass. Surprisingly the fit - although performed for pion masses $600 \ldots 950$ $\mathrm{MeV}$ ! - suggests a quark-mass dependence for the isovector anomalous magnetic moment of the Nucleon which correctly extrapolates down to $\kappa_{v}^{L O}=3.7$

\footnotetext{
${ }^{b}$ We note that even the new set of one-loop diagrams given in Fig.2 obtained to LO in the (modified) Small Scale Expansion contains fewer diagrams than considered in the pioneering one-loop calculation of the haryon magnetic moments by Jenkins et al.8. The 3 additional diagrams considered in Ref. coming in at NLO (i.e. $\mathcal{O}\left(\epsilon^{4}\right)$ ) in our approach, as dictated by the SSE power-counting.
} 
at the physical pion mass of $m_{\pi}=140 \mathrm{MeV}$ ! Such a stable result obtained from only leading order input could not be expected, especially if one compares it with the corresponding HBChPT calculation (dotted curve). Details of the new calculation, including an error analysis, will be available soont. Here I, only want to point out that the analysis suggests that the isovector anomalous magnetic moment has the chiral limit value $\kappa_{v}^{0} \sim 5.7$ [n.m.], which is more than $50 \%$ larger than the value for finite up, down quark masses. This dramatic reduction of the anomalous magnetic moment is mainly due to pionloop effects. Furthermore, one can learn from Fig.1 that the leading linear result of Eq. 1 looses its range of validity already for values smaller than the physical pion mass when curvature effects come in. This finding is consistent with a chiral extrapglation of the same lattice QCD points analyzed with a Pade approximation 10 . Finally, I want to note that the isoscalar anomalous magnetic moment of the Nucleon shows quite a different/much simpler chiral behavior when calculated to the same order in SSE:

$$
\kappa_{s}^{L O}=\kappa_{s}^{(0)}-\frac{E_{2} M_{N}}{4 \pi^{2} F_{\pi}^{2} \Delta} m_{\pi}^{2}+\mathcal{O}\left(\epsilon^{4}\right)
$$

where $\kappa_{s}^{0}$ corresponds to the chiral limit value and $E_{2}$ denotes another unknown $\gamma N N$ coupling; the 2 parameters can also be fitted to isoscalar lattice data, though with larger error bars due to the overall smallness of $\kappa_{s}$.

\section{Isovector Form Factors of the Nucleon}

A few years ago, the role of explicit Delta(1232) degrees of freedom in calculations of the Nucleon form factors at low four-momentum transfer (i f. $\left.Q^{2}<0.2 \mathrm{GeV}^{2}\right)$ was analyzed within the Small Scale Expansion of Ref Considering the discussion in the previous section, one can now ask the question how the results are changed (to leading one-loop order) if one allows for the rescaling of the leading $\gamma N \Delta$-transition as defined in Eq.2, which leads to the two additional (i.e. the last two) Feynman diagrams at $\mathcal{O}\left(\epsilon^{3}\right)$ in Fig.2 and to such a dramatic change in the LO chiral behavior of the isovector anomalous magnetic moment as shown in Fig.1. However, it turns out that to leading order the momentum-dependence of the SSE curves of Ref.6 is not modified by the additional diagrams for a physical pion mass $m_{\pi}=140 \mathrm{MeV}$. The results for the isovector Dirac and Pauli form factor of the Nucleon are shown in Fig. 3. The Dirac form factor turns out to be completely dominated by the radius in this momentum-range. Both the $\operatorname{HBChPT}\left(\mathcal{O}\left(p^{3}\right)\right.$, dot-dashed curve) and the (modified) SSE calculation $\left(\mathcal{O}\left(\epsilon^{3}\right)\right.$, full curve), as well as an empirical parameterization 11 of the data (dashed lines) cannot be distinguished.

NSTAR01: submitted to World Scientific on November 19, 2018 


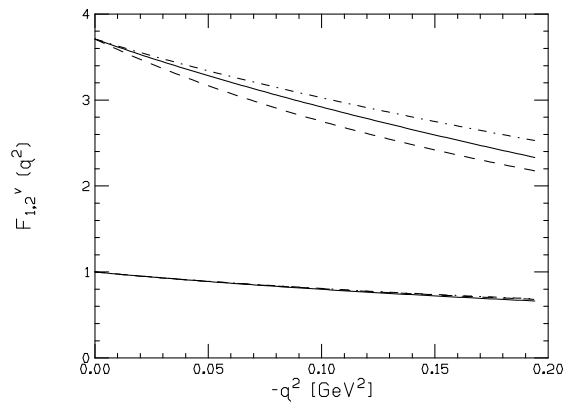

Figure 3. Isovector Dirac and Pauli form factor of the Nucleon, calculated to leading one-loop order with (full curve) and without (dot-dashed curve) explicitppin 3/2 degrees of freedom. Also shown for comparison is a parameterization of the data 11 (dashed curve).

Matters are different for the isovector Pauli form factor. Both the HBChPT and the SSE curves drop slower than suggested by the empirical parameterization. However, the LO SSE calculation arising from the diagrams of Fig.2 provides an isovector Pauli radius of $0.61 \mathrm{fm}^{2}$ - arising solely from the pioncloud around a spin $1 / 2$ or spin $3 / 2$ intermediate baryon - which amounts to more than $75 \%$ of the physical isovector Pauli radius and therefore leads to a qualitatively better description of this form factor than provided by the LO HBChPT calculation of Ref.] (cf. Fig. [3). It will be interesting to study the HBChPT-SSE comparison of the form factors also at NLO, to detemine how far in $q^{2}$ one can trust/fine-tune chiral calculations at the one-loop level.

\section{Isovector Nucleon-Delta Transition Form Factors}

Having discussed the impact of explicit Delta(1232) degrees of freedom in microscopic calculations of the (iso-) vector Nucleon current, one can now address the analogous problems in the isovector Nucleon-Delta trapsition current. This issue has already been analyzed within SSE in Ref.62. Again, the rescaling of the leading order $\gamma N \Delta$ transition vertex of Eq.2 leads to two additional diagrams (displayed in the last row of Fig., (4), compared to the original calculation of Ref.12. However, the additional diagrams only lead to a modified chiral behqzior (i.e. a modified quark mass-dependence of the $\gamma N \Delta$ transition moments $]$ ), whereas the leading order four-momentum dependence for a physical pion-mass of $m_{\pi}=140 \mathrm{MeV}$ of the 3 isovector Nucleon-Delta transition form factors is unchanged. As discussed in Ref.12, microscopic calculations of the $N \Delta$-transition using chiral effective field theories suffer (at 


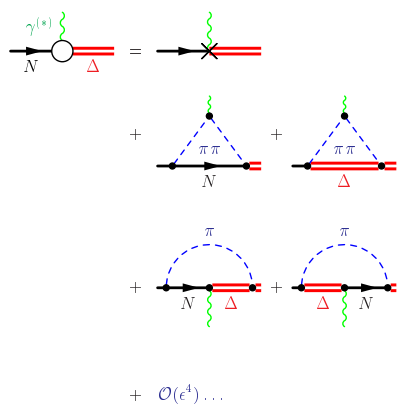

Figure 4. Diagrams contributing to the isovector Nucleon-Delta transition form factors to leading one-loop order in the modified Small Scale Expansion.

present) from the fact that there are several unknown counter-terms, which need to be fixed via external input. As an example, in Fig. . called CMR, i.e. the ratio of the $\mathrm{C} 2$ over the M1 Nucleon-Delta transition strength as a function of the four-momentum transfer $q^{2}$. Depending on different ways of fixing the counter-term input as described in 12 , one obtains a rather broad band of possible momentum-dependence for the CMR ? The most promising way to improve upon these theoretical limitations consists of a full calculation of the pion-electroproduction cross-section in the resonance region within a chiral effective field theory like SSE. So far, this has not been attempted d due to the high order required for an adequate treatment of the width of the Delta resonance. It is interesting to note that the proposed rescaling of the leading $\gamma N \Delta$ transition vertex Eq.2 also provides new hope to get this longstanding problem in chiral effective field theory calculations finally done, as in the modified SSE the order required to include these width effects is lowered; first exploratory studies are under way.

\section{Summary}

I have given a report regarding some new ideas/open problems in the field of chiral effective field theories with explicit spin $3 / 2$ resonance degrees of freedom. I am convinced that the role and the treatment of baryon resonances continues to be an inspiring and challenging topic in this field.

${ }^{c}$ For the status of experimental information on the momentum dependence of CMR I refer to the talks by R.W. Gothe, G.N Papanicolas, and H. Schmieden in these proceedings.

${ }^{d}$ An exception is work of Ref.13, where the authors tried to utilize a low order SSE calculation in the resonance region by averaging over the width of the resonance. 


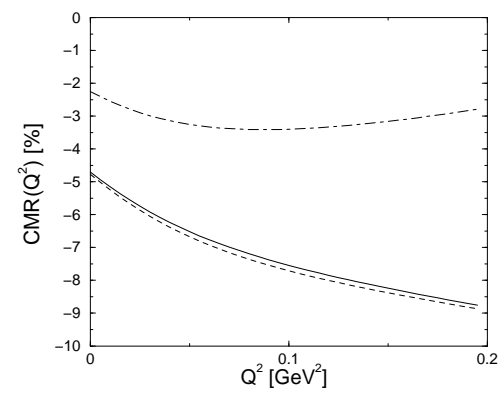

Figure 5. Real part of the four-momentum dependence of the ratio of the C2/M1 $\gamma N \Delta$ transition moments, calculated to leading one-loop order in the Small Scale Expansion. The spread of the curves is discussed in the text.

\section{Acknowledgments}

I want to thank the organizers of NSTAR01 for giving me the opportunity to present these results to the community and I gratefully acknowledge their financial support. I also would like to thank A. Thomas and W. Weise for helpful discussions. The research presented here was supported by BMBF.

\section{References}

1. see e.g. U.-G. Meißner, preprint no. [hep-ph/0007092].

2. H.W. Grießhammer and G. Rupak, preprint no. [hucl-th/0012096].

3. see e.g. T.R. Hemmert, preprint no. nucl-th/0101054].

4. T.R. Hemmert, B.R. Holstein and J. Kambor, J. Phys. G24 (1998) 1831; Phys. Lett. B395 (1997) 89.

5. V. Bernard et al., Nucl. Phys. B388, 315 (1992).

6. V. Bernard et al., Nucl. Phys. A635, 121 (1998).

7. T.R. Hemmert and W. Weise, forthcoming.

8. E. Jenkins et al., Phys. Lett. B302, 482 (1993).

9. D.B. Leinweber, R.M. Woloshyn and T. Draper, Phys. Rev. D43, 1659 (1991).

10. A.W. Thomas, these proceedings; see also D.B. Leinweber, D.H. Lu and A.W. Thomas, Phys. Rev. D60, 034014 (1999) and references therein.

11. P. Mergell, U.-G. Meißner and D. Drechsel, Nucl. Phys. A596, 367 (1996).

12. G.C. Gellas et al., Phys. Rev. D60, 054022 (1999).

13. C.W. Kao and T.D. Cohen, Phys. Rev. C60, 064619 (1999). 